\title{
Mesenchymal stem cells are efficiently transduced with adenoviruses bearing type 35-derived fibers and the transduced cells with the IL-28A gene produces cytotoxicity to lung carcinoma cells co-cultured
}

\author{
Takeo Suzuki ${ }^{1,2}$, Kiyoko Kawamura², Quanhai Li ${ }^{2,3}$, Shinya Okamoto ${ }^{2,4}$, Yuji Tada $^{4}$, Koichiro Tatsumi ${ }^{4}$, \\ Hideaki Shimada ${ }^{5}$, Kenzo Hiroshima ${ }^{6}$, Naoto Yamaguchi ${ }^{1}$ and Masatoshi Tagawa ${ }^{2,3^{*}}$
}

\begin{abstract}
Background: Transduction of human mesenchymal stem cells (MSCs) with type 5 adenoviruses (Ad5) is limited in the efficacy because of the poor expression level of the coxsackie adenovirus receptor (CAR) molecules. We examined a possible improvement of Ad-mediated gene transfer in MSCs by substituting the fiber region of type 5 Ad with that of type $35 \mathrm{Ad}$.

Methods: Expression levels of CAR and CD46 molecules, which are the major receptors for type 5 and type 35 Ad, respectively, were assayed with flow cytometry. We constructed vectors expressing the green fluorescent protein gene with Ad5 or modified Ad5 bearing the type 35 fiber region (AdF35), and examined the infectivity to MSCs with flow cytometry. We investigated anti-tumor effects of MSCs transduced with interleukin (IL)-28A gene on human lung carcinoma cells with a colorimetric assay. Expression of IL-28A receptors was tested with the polymerase chain reaction. A promoter activity of transcriptional regulatory regions in MSCS was determined with a luciferase assay and a tumor growth-promoting ability of MSCs was tested with co-injection of human tumor cells in nude mice.

Results: MSCs expressed CD46 but scarcely CAR molecules, and subsequently were transduced with AdF35 but not with Ad5. Growth of MSCs transduced with the IL-28A gene remained the same as that of untransduced cells since MSCs were negative for the IL-28A receptors. The IL-28A-transduced MSCs however suppressed growth of lung carcinoma cells co-cultured, whereas MSCs transduced with AdF35 expressing the $\beta$-galactosidase gene did not. A regulatory region of the cyclooygenase-2 gene possessed transcriptional activities greater than other tumor promoters but less than the cytomegalovirus promoter, and MSCs themselves did not support tumor growth in vivo.

Conclusions: AdF35 is a suitable vector to transduce MSCs that are resistant to Ad5-mediated gene transfer. MSCs infected with AdF35 that activate an exogenous gene by the cytomegalovirus promoter can be a vehicle to deliver the gene product to targeted cells.
\end{abstract}

Keywords: Mesenchymal stem cells, Adenovirus, Type 35 adenovirus fiber, IL-28A

\footnotetext{
* Correspondence: mtagawa@chiba-cc.jp

${ }^{2}$ Division of Pathology and Cell Therapy, Chiba Cancer Center Research Institute, Chiba, Japan

${ }^{3}$ Department of Molecular Biology and Oncology, Graduate School of Medicine, Chiba University, Chiba, Japan

Full list of author information is available at the end of the article
} 


\section{Background}

Bone marrow-derived mesenchymal stem cells (MSCs) have ability to differentiate into many kinds of tissues under a certain condition [1,2]. The pluripotency as progenitor cells indicates a potential clinical utility in multiple areas including tissue engineering. Furthermore, MSCs tend to migrate into inflammatory regions, damaged areas and tumors [3,4], which increases applications of MSCs as a tool to deliver an agent to target tissues and cells. Gene and cell therapy can be one of the directions to use MSCs as a cellular vehicle that distumors. Administration of transduced MSCs in the vicinity of tumors can transport the gene product into the microenvironment as well.

Adenoviruses (Ad)-mediated transduction is one of the efficient methods to transfer an exogenous gene into human cells. The transduction efficacy with Ad vectors is however influenced by expression levels of the receptor molecules on target cells [5]. Attachment of type 5 Ad (Ad5), commonly used in a gene transfer system, to cells is mediated primarily by the binding of Ad fibers, which include the shaft and the knob regions, to the cellular receptor, the coxsackie adenovirus receptor (CAR) molecules, and secondly by the interaction between Ad penton bases and integrin molecules [6]. Expression levels on CAR molecules are dependent on respective cells and are often down-regulated in human tumors, which resulted in poor transduction efficacy in CAR-low cells [7]. On the other hand, subgroup B Ad such as type 35 use CD46 molecules as one of the major receptors and infect cells in a CAR-independent manner [8]. CD46 is expressed in a variety of human cells and the expression levels were not down-regulated in tumors. Recombinant Ad5 of which the fiber region is replaced with that of type 35 Ad (AdF35) can therefore infect cells in a similar manner as type 35 Ad through the type 35-derived fibers, which may widen a scope of target cells that are restricted by non-ubiquitous CAR distributions.

A new class of interferon (IFN), type III IFNs comprising of IFN- $\lambda 1,-\lambda 2$ and $-\lambda 3$ which are also known as interleukin-29 (IL-29), IL-28A and IL-28B, respectively, has a similar biological functions as type I IFNs such as IFN- $\alpha$ and IFN $-\beta[9,10]$. The receptor complex of type III IFNs is composed of the IL-10 receptor beta (IL-10R $\beta$ ) and a novel IL-28 receptor alpha (IL-28R $\alpha$ ). In contrast to ubiquitous expression of IL-10R $\beta$, IL-28R $\alpha$ expression is restricted to be tissue-specific, which subsequently confines the biological activities in IL-28R $\alpha$ positive cells. The type III IFNs produce an anti-proliferative activity and induce apoptosis to a certain type of the receptor positive tumors including lung carcinoma [11] and esophageal carcinoma [12]. Moreover, several studies demonstrated that type III IFNs expressed in tumors achieved anti-tumor effects in vivo and some of the effects were mediated by non-immune mechanisms including anti-angiogenesis and by immunological responses such as activation of natural killer cells and dendritic cells [13-17].

In this study, we examined infectivity of Ad5 and AdF35 to human MSCs and investigated a possible use of MSCs as a vehicle to deliver gene products to tumors. We transduced MSCs with the IL-28A gene using a replication-incompetent AdF35 vector and tested whether the transduced MSCs produced cytotoxicty to tumor cells co-cultured. We also examined promoter activities in MSCs regarding transcriptional regulatory regions of the genes which are preferentially activated in human tumors.

\section{Methods \\ Cells and mice}

Human embryonic kidney HEK293 cells, human esophageal carcinoma YES-2 and TE-11 cells, human lung carcinoma OBA-LK1 cells, human immortalized fibroblasts OUMS-24 [18] and HFF cells [19], were cultured with RPMI1640 cells supplemented with $10 \%$ fetal bovine serum. MSCs derived from human bone marrow (PT-2501) (Cambrex, Rutherford, NJ, USA) were maintained with Mesenchymal Stem Cell Basal Medium (MSCBM; Cambrex). BALB/c nu/nu mice were purchased from Japan SLC (Hamamatsu, Japan).

\section{Flow cytometry for receptor expression}

Cells were stained with fluorescein isothiocyanate (FITC)conjugated anti-CD46 antibody (Ab) (BD Bioscience, San Jose, $\mathrm{CA}$ ) or FITC-conjugated isotype-matched control $\mathrm{Ab}$ (BD Biosciences) as a control, or were reacted with anti-CAR (Upstate, Lake Placid, NY, USA), anti-CD51 (Chemicon, Temecula, CA, USA), anti- $\alpha v \beta 3$ (Chemicon) or anti- $\alpha v \beta 5 \mathrm{Ab}$ (Abcam, Cambridge, MA, USA) followed by FITC-conjugated goat anti-mouse IgG Ab (Kirkegaard \& Perry, Gaithersburg, MD, USA). They were then analyzed for the fluorescence intensity with FACSCalibur (BD Bioscience) and CellQuest software (BD Bioscience).

\section{Construction of Ad vector}

The green fluorescent protein (GFP), the $\beta$-galactosidase (LacZ), the human $I L-28 A$ genes were cloned into pShuttle 2 (Takara Bio, Tokyo, Japan) and then ligated with Adeno-X vector (Takara Bio) of which the fiber region was replaced with that of type $35 \mathrm{Ad}$. The fiber modified Ad DNA was produced by inserting the Eco RI fragment containing the type 35 Ad fiber region (Avior therapeutics, Seattle, WA) (AY271307 at 30827-33609) into the corresponding site of Adeno-X vector DNA. The fiber modified Ad expressing the above genes, AdF35-GFP, AdF35-LacZ, and AdF35-IL-28A, and type 5 Ad bearing the GFP gene (Ad5-GFP) were produced by transfecting 
the respective DNA into HEK293 cells and purified with an Adeno-X virus purification kit (BD Biosciences).

\section{Infectivity of Ad}

Cells were infected with Ad5-GFP or AdF35-GFP at multiplicity of infection (MOI) of 3 or 30 for $30 \mathrm{~min}$ and were washed to remove Ad. Infected cells were cultured for 2 days and then analyzed for percentages of GFPpositive cells with FACSCalibur and CellQuest software. Cells of which fluorescence was greater than the brightest $5 \%$ of uninfected cells were judged as positively stained.

Reverse transcription-polymerase chain reaction (RT-PCR) First-strand cDNA was synthesized with Superscript III reverse transcriptase (Invitrogen, Carlsbad, CA) and amplification of equal amounts of the cDNA was performed with the following primers and conditions: for the $I L-28 R \alpha$ gene, 5'-GGGAACCAAGGAGCTGCTAT G-3' (sense) and 5'-TGGCACTGAGGCAGTGGTGT $\mathrm{T}-3^{\prime}$ (anti-sense), and $10 \mathrm{sec}$ at $94^{\circ} \mathrm{C}$ for denature/20 sec at $58^{\circ} \mathrm{C}$ for annealing $/ 28$ cycles; for the $I L-10 R \beta$ gene, 5'-TATTGGACCCCCTGGAAT-3' (sense) and 5'-GTA AACGCACCACAGCAA-3' (anti-sense), and $10 \mathrm{sec}$ at $94^{\circ} \mathrm{C} / 20 \mathrm{sec}$ at $50^{\circ} \mathrm{C} / 28$ cycles; for the glyceraldehyde3-phosphate dehydrogenase (GAPDH) gene, 5'-ACCAC AGTCCATGCCATCAC-3' (sense) and 5'-TCCACCAC CCTGTTGCTGTA-3' (anti-sense), and $15 \mathrm{sec}$ at $94^{\circ} \mathrm{C} /$ $15 \mathrm{sec}$ at $60^{\circ} \mathrm{C} / 25$ cycles.

\section{Cytotoxic test and enzyme-linked immunosorbent assay (ELISA)}

OBA-LK1 cells were cultured in 96-well plates with MSCs uninfected or infected with AdF35-IL-28A or AdF35-LacZ (MOI $=100)$, at a ratio of 10: 1 or 10: 3 . Cell viabilities after 4-days culture were assayed with a WST kit (Dojindo, Kumamoto, Japan) which detected the amounts of formazan produced from the WST-8 reagent with the absorbance at $450 \mathrm{~nm}$ (WST assay). OBA-LK1 cells were also labeled with PKH 26 dye according to the manufacturer's protocol (Sigma-Aldrich, St Louis, MO, USA) and cultured with MSCs, uninfected or infected with AdF35-IL-28A or AdF35-LacZ $(\mathrm{MOI}=100)$, at a ratio of 10: 1 or 10: 3 for 4 days. They were then stained with Hoechst 33342 dye (Molecular Probes, Eugene, OR, USA) and numbers of PKH 26 positive and Hoechst 33342 positive cells were counted with confocal microscope (Olympus, Tokyo, Japan). The amounts of secreted IFN-28A were determined by an ELISA kit (R\&D Systems, Minneapolis, MN).

\section{Dual luciferase assay}

Genomic fragments containing a transcriptional regulatory region of the midkine (0.6 kb, GenBank: D10604) [20], the survivin (0.5 kb, GenBank: U75285) [21], or the cyclooxygenase-2 (0.3 kb, GenBank: U04636) gene [22] were cloned into pGL-2 basic vector (Promega, Madison, WI, USA) that contained the firefly luciferase gene. Plasmid DNA containing the respective genomic fragments, pGL-control vector (Promega) harboring the SV40 T antigen promoter-linked firefly luciferase gene, pGL-2 basic vector containing the cytomegalovirus (CMV) promoter or pGL-basic vector without any transcriptional regulatory regions (Promega), and a control vector, the renilla luciferase gene fused with the herpes simplex virus-thymidine kinase gene promoter (pRL-TK, Promega), at a molar ratio of 10: 1, was transfected into MSCs with a lipofectin reagent (Life Technologies, Gaithersburg). Cell lysate on day 2 was assayed for the luciferase activity with the dual luciferase reporter assay (Promega). The firefly luciferase activity was standardized by the amounts of luminescence produced by renilla luciferase and the relative activity was expressed as a percentage of the SV40 T antigen promoter-mediated activity.

\section{Animal study}

YES-2 cells $\left(1 \times 10^{6}\right)$ and MSCs or OUMS-24 cells at a ratio 5: 1 or 2 : $1\left(2 \times 10^{5}\right.$ or $\left.5 \times 10^{5}\right)$ were inoculated subcutaneously into BALB/c nu/nu mice (6-week-old females). Tumor volume was calculated according to the formula $\left(1 / 2 \times\right.$ length $\mathrm{x}$ width $\left.{ }^{2}\right)$.

\section{Statistical analysis}

Statistical analysis was conducted with the one-way analysis of variance (ANOVA).

\section{Results}

\section{Expression of Ad receptors on MSCs}

We examined expression levels of CAR and CD46 molecules, the major Ad receptors of type 5 and type 35, respectively, on HEK293 cells and MSCs (Figure 1A). HEK293 cells, often used for Ad productions, expressed both receptors, whereas MSCs scarcely expressed CAR but were positive for CD46 expression. We also tested integrin molecules which were subsidiary receptors for type 5 Ad (Figure 1B). Both HEK293 cells and MSCs expressed CD51 that corresponded to integrin $\alpha v$ chain, $\alpha v \beta 3$ and $\alpha v \beta 5$ molecules. We calculated relative expression levels of these receptor molecules on MSCs in comparison with HEK293 cells and showed that MSCs expressed poorly CAR, moderately CD46 and well integrin molecules (Figure 2).

\section{Infectivity of Ad5 and AdF35 to MSCs}

We investigated efficacy of Ad5- and AdF35-mediated transduction with respective Ad bearing the GFP gene (Figure 3A). HEK293 cells became GFP positive after transduction with either Ad5-GFP or AdF35-GFP, but MSCs expressed GFP only when transduced with AdF35GFP (Figure 3B). Percentages of GFP positive HEK293 


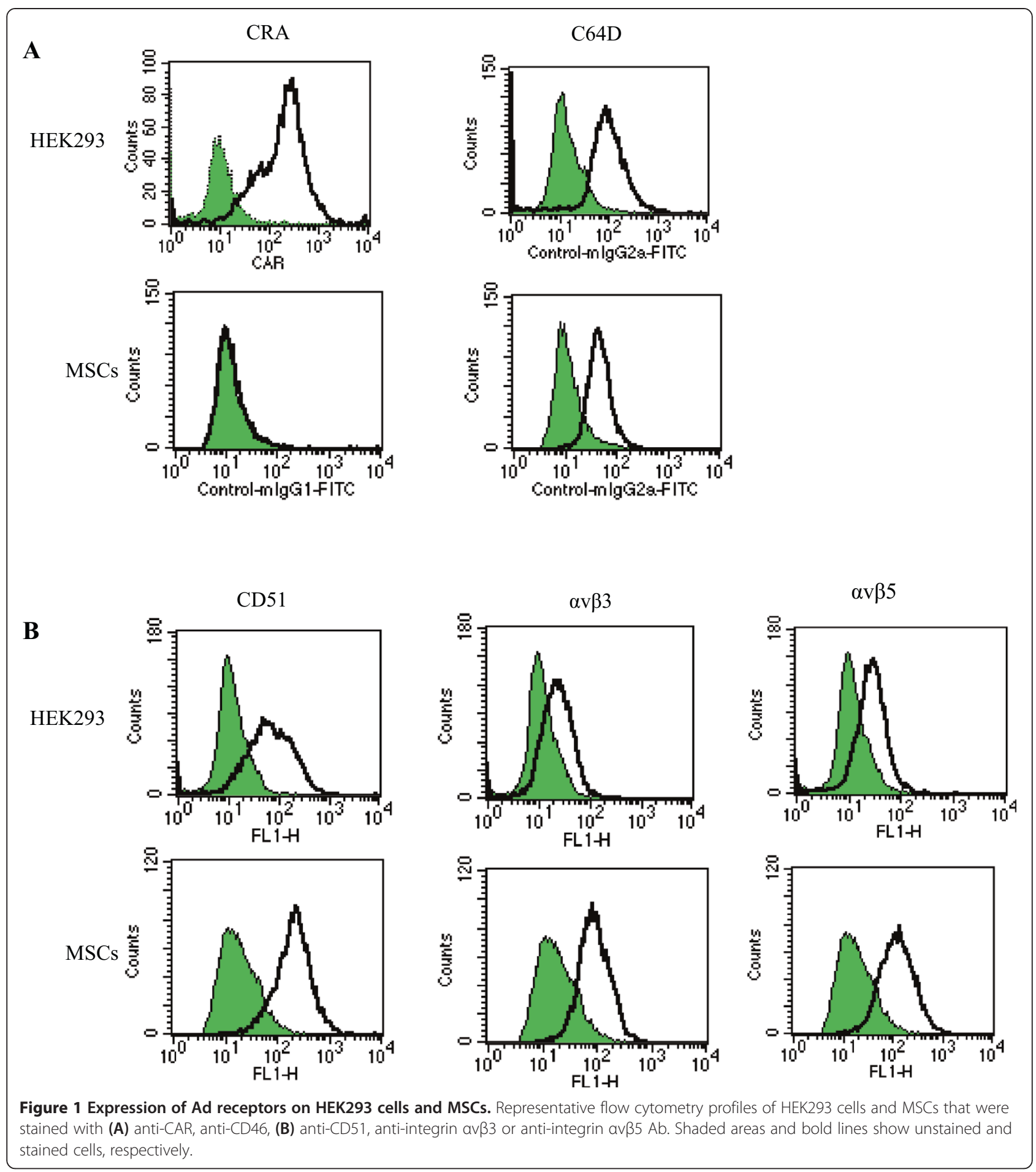

cells were not different when they were transduced either with Ad5-GFP or AdF35-GFP (Figure 4). In contrast, GFP positive MSCs cells were undetected with Ad5-mediated transduction and the positive percentages after transduction with AdF35-GFP were lower than those of HEK293 cells. The differential GFP positive rates were attributable to viral infectivity to the cells since both Ad5-GFP and AdF35-GFP used the same CMV promoter.

Anti-tumor effects of MSCs infected with AdF35-IL-28A IL-28A produced cytotoxic effects on cells expressing the receptor complex, IL-28R $\alpha$ and IL-10R $\beta$. We examined 


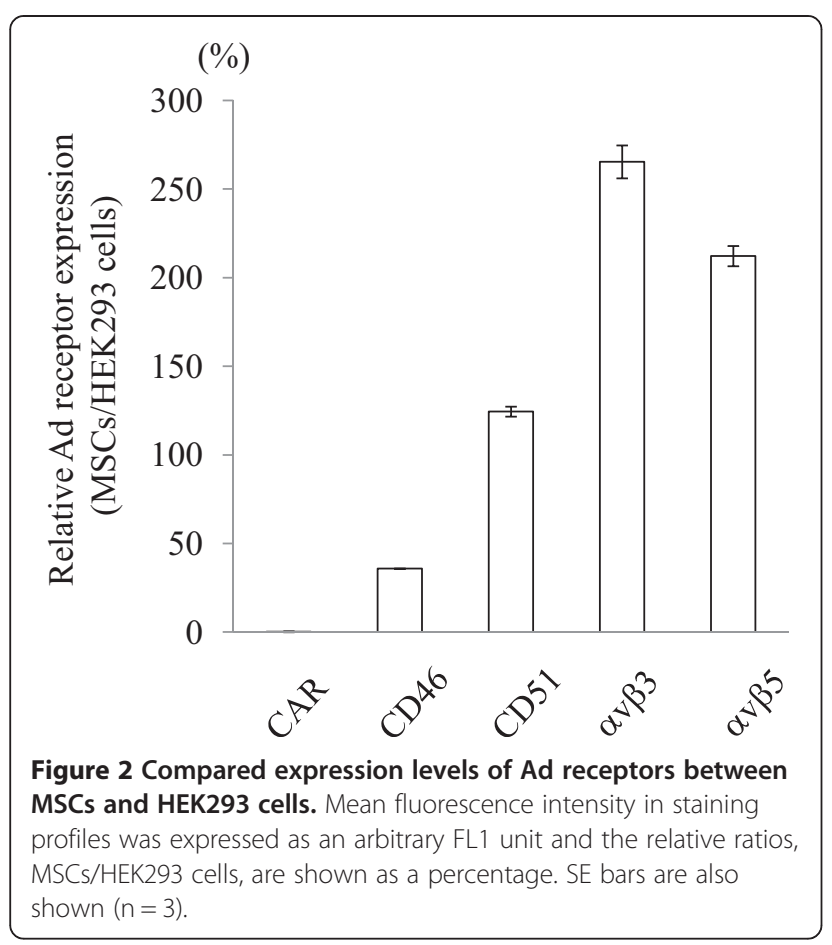

the expression on MSCs together with immortalized fibroblasts, OUMS-24 and HFF cells, and esophageal carcinoma TE-11 cells as a reference of normal cells and as a positive control for the IL-28A receptor complex, respectively (Figure 5A) [12]. MSCs and the fibroblasts expressed IL-10R $\beta$ but not IL-28R $\alpha$, demonstrating that MSCs were insensitive to IL-28A. In contrast, the esophageal carcinoma cells were positive for both molecules [12].

We then examined possible cytotoxicity of IL-28A released from MSCs in a co-culture experiment. Lung carcinoma OBA-LK1 cells were positive for the IL-28A receptor complex and the growth was suppressed by recombinant IL-28A [11]. We infected MSCs with AdF35IL-28A or AdF35-LacZ as a control and detected IL-28A released from MSCs with ELISA at $363 \pm 4.61 \mathrm{pg} / \mathrm{ml} /$ day per $10^{3}$ MSCs. IL-28A-sensitive OBA-LK1 cells were mixed with the MSCs and the viable cell numbers were estimated with the WST assay (Figure 5B). Viability of MSCs that were either uninfected or infected with AdIL-28A or Ad-LacZ was not statistically different, showing that expression of IL-28A did not induce growth suppression in MSCs. In contrast, absorbance of a mixed population consisting of OBA-LK1 cells and AdF35-IL28A-infected MSCs was lower than that of a mixture of OBA-LK1 cells and either uninfected MSCs or AdF35LacZ-infected MSCs. Absorbance of the cell mixture of

A

\begin{tabular}{rl|l|l|c|c|}
\multicolumn{3}{c}{ E1 region } & \multicolumn{2}{c}{ Fiber } \\
\cline { 2 - 6 } Ad5-GFP & CMV-GFP & & Type 5 & \\
\cline { 2 - 6 } AdF35-GFP & & CMV-GFP & & Type 35 & \\
\cline { 2 - 6 } & & &
\end{tabular}

B

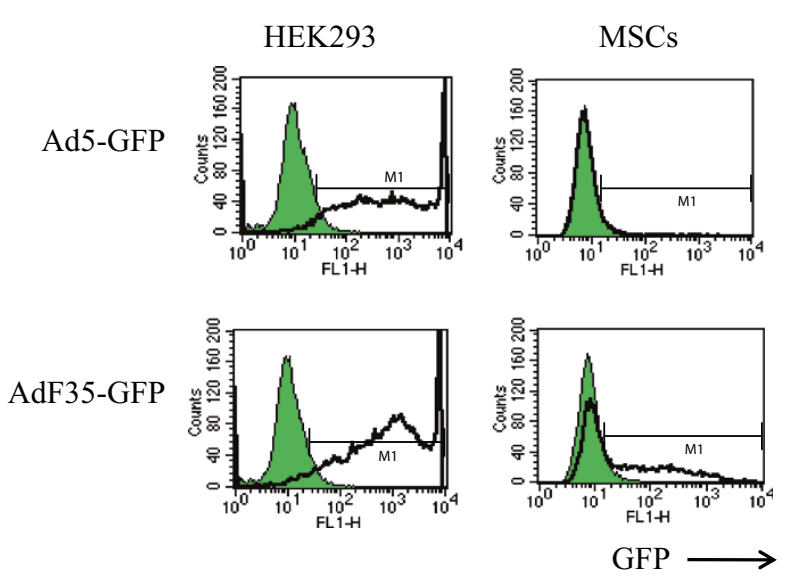

Figure 3 Infectivity of Ad to MSCs. (A) Schematic structures of Ad5-GFP and AdF35-GFP. The E1 region was replaced with the CMV promoter-linked GFP gene. (B) Representative flow cytometry profiles of HEK293 cells and MSCs that were infected with Ad5-GFP or AdF35-GFP. M1 indicates positively stained population, and shaded areas and bold lines show uninfected and infected cells, respectively. 


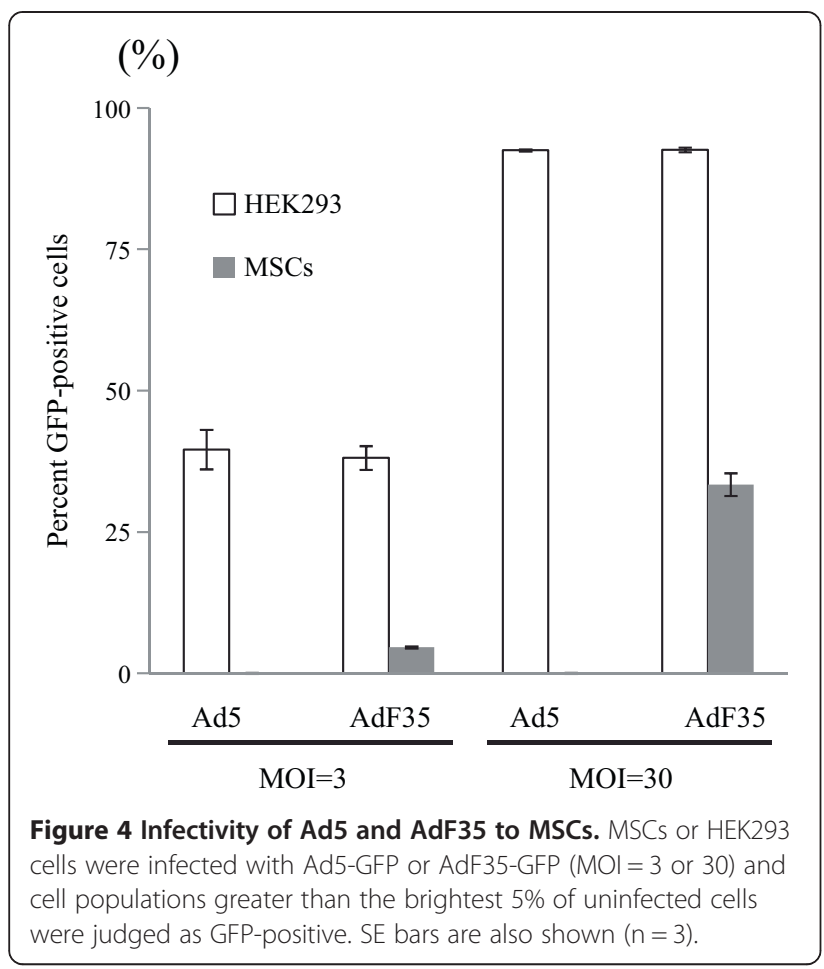

OBA-LK1 cells and AdF35-IL-28A-infected MSCs was even lower than that of OBA-LK1 cells alone, indicating that IL-28A released from MSCs inhibited growth of OBA-LK1 cells.

We confirmed growth inhibitory activities of MSCs transduced with AdF35-IL-28A in a different assay (Table 1). OBA-LK1 cells were labeled with PKH 26 and cultured with MSCs for 4 days. We then stained all the cells with Hoechst 33342 and calculated numbers of PKH 28 positive cells among Hoechst 33342 positive cells. Percentages of PKH 28 positive OBA-LK1 cells were about $80 \%$ because the PKH 28 labeling was not complete under the experimental condition. OBA-LK1 cells cultured with untransduced MSCs further decreased the $\mathrm{PKH} 28$ positive ratio since $\mathrm{PKH} 26$ negative MSCs were also counted. Percentages of PKH 26 positive cells in cell mixtures were not different between co-culture with uninfected MSCs and that with AdF35LacZ-infected MSCs irrespective of a ratio of the mixtures. The percentages however significantly lower in co-culture with AdF35-IL-28A-infected MSCs compared with cell mixture with uninfected MSCs or AdF35LacZ infected MSCs. These data demonstrated that MSCs-derived IL-28A inhibited growth of OBA-LK1 cells.

Transcriptional regulation and growth assistance in MSCs We investigated whether a putative tumor promoter could activate the luciferase gene in non-tumorous MSCs. We therefore examined transcriptional regions of the midkine, the survivin and the $C O X-2$ genes for the promoter

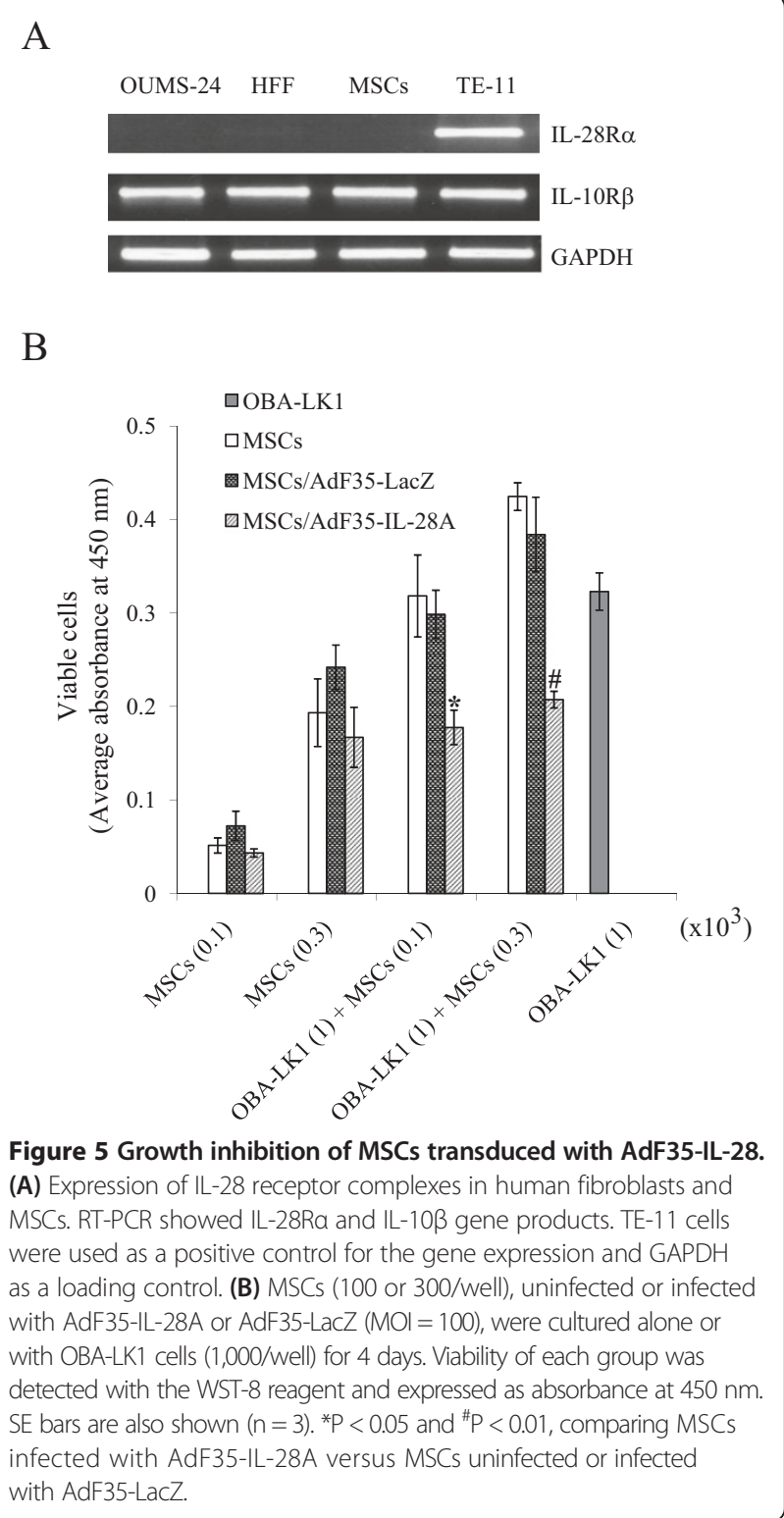

activity in MSCs with the SV40 T antigen promoter region as a reference (Table 2). These regions are often used for activation of a transgene in a tumor-specific manner. A regulatory region of the $C O X-2$ gene activated the luciferase gene greater than that of the midkine or the survivin gene. A transcriptional activity of the COX region was greater than that of the SV40 T antigen promoter, but much less than that of the CMV promoter which is commonly used for transgene activations in many cells.

We also examined a possible tumor growth-promoting activity of MSCs with animal experiments (Figure 6). We inoculated YES-2 esophageal carcinoma cells into nude mice without or with MSCs or fibroblasts OUMS24. Tumor growth of mixed populations, irrespective of 
Table 1 Growth suppression of OBA-LK1 cells cultured with transduced MSCs

\begin{tabular}{llll}
\hline OBA-LK1 & $\begin{array}{l}\text { MSCs infected } \\
\text { with }\end{array}$ & $\begin{array}{l}\text { Mixed cell ratio } \\
\text { (OBA-LK1 : MSCs) }\end{array}$ & $\begin{array}{l}\text { PKH 26 positive cells } \\
\text { (Percentage } \pm \text { SE) }^{1}\end{array}$ \\
\hline$(+)$ & $(-)$ & & $80.2 \pm 2.0$ \\
$(+)$ & None & $10: 1$ & $57.4 \pm 4.2^{*}$ \\
$(+)$ & AdF35-LacZ & $10: 1$ & $65.6 \pm 4.1^{*}$ \\
$(+)$ & AdF35-IL28A & $10: 1$ & $47.6 \pm 2.7^{*}$ \\
$(+)$ & None & $10: 3$ & $67.0 \pm 4.4^{\#}$ \\
$(+)$ & AdF35-LacZ & $10: 3$ & $56.1 \pm 2.0^{\#}$ \\
$(+)$ & AdF35-IL-28A & $10: 3$ & $26.4 \pm 1.4^{\#}$ \\
\hline
\end{tabular}

OBA-LK1 cells $\left(3.3 \times 10^{4}\right)$ stained with PKH 26 were cultured with MSCs at a ratio indicated, and all the cells were then stained with Hoechst 33342 on day 4. ${ }^{1}$ Percentages of PKH 26 positive cells among Hoechst 33342 positive cells and SEs are also shown $(n=3)$.

${ }^{*} \mathrm{P}<0.05$ and ${ }^{*} \mathrm{P}<0.01$, comparing between cells mixed with AdF35-IL-28Ainfected MSCs and cells mixed with uninfected MSCs or AdF35-LacZ-infected MSCs.

the ratio or mixed cells, was not different from that of YES-2 cells alone, demonstrating that MSCs, like fibroblasts, did not support tumor growth of YES-2 esophageal carcinoma cells co-injected.

\section{Discussion}

We showed that MSCs were resistant to Ad5-mediated gene transfer but were transduced with fiber-modified AdF35 vectors. The transduction preference was linked with the Ad receptor expression on MSCs, which were negative for CAR but positive for CD46 molecules. Comparison of receptor expressions between HEK293 cells and MSCs further showed that CD46 expression levels on MSCs were not as great as those on HEK293 cells, which resulted in lower transduction efficacy of AdF35 to MSCs than to HEK293 cells. Interestingly, expression levels of $\alpha v \beta 3$ and $\alpha v \beta 5$ integrin molecules were

Table 2 Promoter activity of transcriptional regulatory regions in MSCs

\begin{tabular}{|c|c|}
\hline $\begin{array}{l}\text { Transcriptional regulatory } \\
\text { region }\end{array}$ & 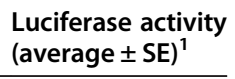 \\
\hline$(-)$ & $16.8 \pm 8.9$ \\
\hline SV40 T antigen & $100.0 \pm 11.5^{*}$ \\
\hline Midkine & $66.8 \pm 27.6^{*}$ \\
\hline Survivin & $47.5 \pm 14.7^{*}$ \\
\hline $\operatorname{cox}-2$ & $368.2 \pm 182.8^{*}$ \\
\hline CMV & $6323.4 \pm 2067.3^{*}$ \\
\hline
\end{tabular}

${ }^{1}$ Relative luciferase activity was calculated based on the SV40 T antigen promoter-mediated activity as $100 \%$. SEs are shown $(n=3)$.

${ }^{*} \mathrm{P}<0.05$, comparing between the COX-2 region and either the SV40 T antigen, the survivin regulatory region, the midkine regulatory region or the CMV promoter. greater on MSCs than on HEK293 cells. Nevertheless, transduction of MSCs with Ad5 was not detected, indicating that the integrin molecules did not play a role as an auxiliary receptor in MSCs although the integrin molecules were demonstrated to be the major receptor of Ad5 in CAR-deficient cells [23]. In addition, the integrin molecules were shown to enhance AdF35-mediated gene transduction [24], but the present study suggested that elevated expression levels of integrin molecules on MSCs cells could not restore transduction efficacy of AdF35 to MSCs to the same level as to HEK293 cells. Many factors seem to be involved in mechanisms underlying Ad infectivity and the mechanisms can be different among cell types tested. They may include a possible threshold level of the receptor expression necessary for Ad infection and a presumable reciprocal interaction among the receptor molecules. CD46 is not a sole receptor for AdF35 and the expression levels were relatively low in freshly isolated MSCs [25]. Nevertheless, the MSCs from adult donors were infected with AdF35 [25], and we presume that Ad vectors bearing the type 35 fiber is currently one of the efficient vectors for gene transfer into MSCs.

Usage of gene modified MSCs has several advantages over direct Ad administrations in the anti-tumor activity. Transduced MSCs, injected intratumorally, tend to localize at the tumor sites in contrast to Ad which are subjected to a rapid washout from the injection sites [26]. Propensity of MSCs to migrate into tumors is thus favorable for MSCsmediated anti-tumor effects [27] although the property was dependent on an experimental system [28]. Administration of MSCs into a tumor site also needs careful consideration since MSCs can promote the tumor growth due to the ability to constitute and maintain the microenvironments around tumors. We thereby examined the possible growth-enhancing activity in vivo and showed that MSCs did not support tumor growth of esophageal carcinoma cells co-injected. MSCs were not able to enhance tumor growth in nude mice but the possibility of tumor promoting actions needs to be studied in different experimental models. On the other hand, the present study showed that untransduced MSCs did not achieve any anti-tumor effects by themselves. The growth suppressing activity of transduced MSCs was thus attributable to IL-28A since the suppression was dependent on cell numbers of $I L-28-$ transduced but not on $\beta$-galactosidase-transduced MSCs. A property of expressed transgene products is also crucial for therapeutic efficacy of MSCs-mediated gene delivery. For example, IL-28A not only induces tumor cell death through apoptosis but activates inmate and acquired immunity through augmented natural killer activities and facilitated antigen presentation [12,14-17]. Moreover, IL-28A in vivo influences and modulates tumor microenvironments such as inhibition of angiogenesis, which can be mediated by other cytokines [13]. 


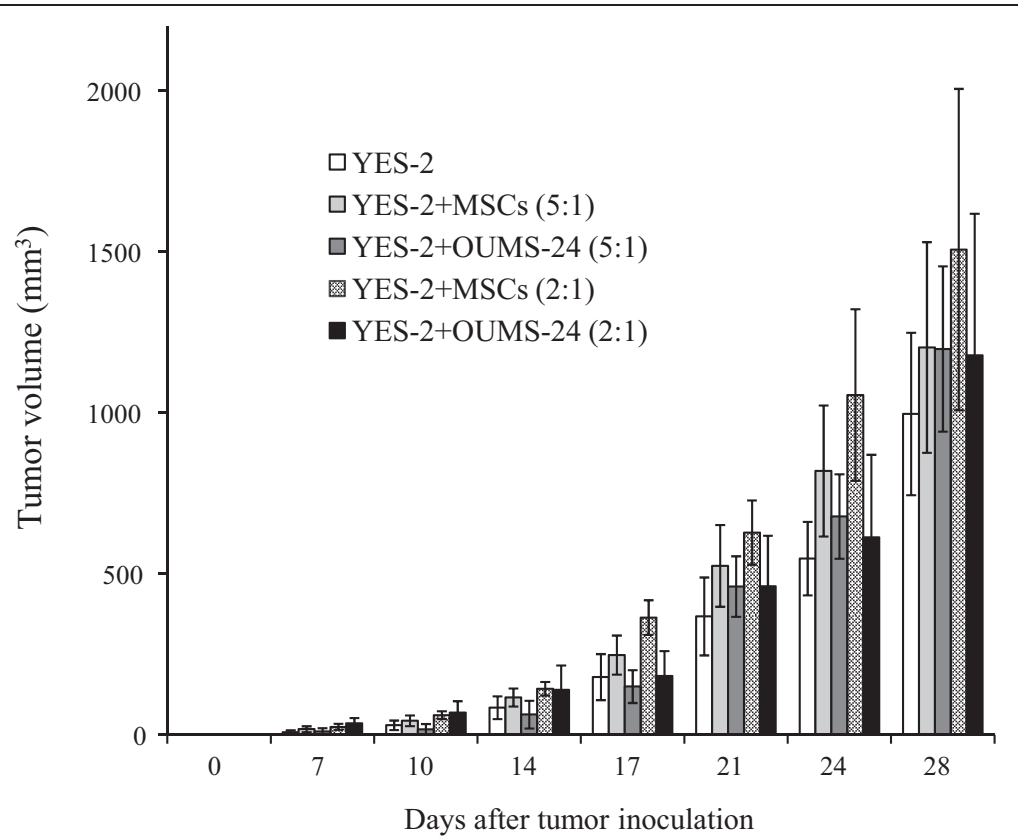

Figure 6 MSCs did not influence growth of co-injected tumors. YES-2 cells and MSCs or OUMS-24 cells were inoculated subcutaneously into $\mathrm{BALB} / \mathrm{C}$ nu/nu mice at the ratios indicated. Average tumor volumes and SE bars are shown $(n=6)$.

Recently, several reports demonstrated anti-tumor effects produced by MSCs-mediated delivery of replicationcompetent Ad into tumors [28,29] and a clinical research revealed benefits of such autologous MSCs in neuroblastoma patients [30]. Tumor cells were initially used for cellmediated delivery of replication-competent Ad [31] since tumor cells well supported Ad replication compared with non-transformed cells. MSCs may not be effective in the light of production of progenitor Ad because of the low proliferation rate. We examined the promoter activity of transcriptional regulatory regions which activated the $A d$ $E 1 A$ gene and subsequently enabled Ad replicationcompetent within tumors [32-34]. The COX-2 region gave greater activities than the midkine or the survivin region. Expression levels of midkine and survivin in adult tissues are often associated with proliferation rates of cells [35] and relatively low promoter activities of these regions could reflect the low growth rates of MSCs. Instead, COX-2 expression can be liked with inflammatory responses [36,37]. Elevated promoter activity of the COX-2 region in MSCs may be related to MSCs' propensity to migrate toward inflammatory sites. Nevertheless, a promoter activity of the $C O X-2$ region was much lower than that of the CMV promoter which is commonly used to activate a transgene in replication-incompetent Ad. These data suggest that MSCs are a suitable cell-mediate vehicle for CMV promoter-driven replication-incompetent Ad rather than for replication-competent Ad in which the E1A is activated by an exogenous transcriptional regulatory region.

\section{Conclusions}

We demonstrated anti-tumor effects of MSCs-mediated delivery of IL-28A to lung carcinoma cells in the vicinity. A local administration of gene-modified MSCs can deliver the gene product to targets and is one of the cell therapies for cancer. AdF35 is a better vector than conventional type $5 \mathrm{Ad}$ in transducing MSCs because of its enhanced infectivity. Immune responses against genemodified MSCs are less significant as long as MSCs are autologously isolated. Nevertheless, when MSCs are infected with replication-competent Ad, cell-mediated immunity against viral gene-loaded MSCs will be generated. Further investigations are required for the cell-mediated immunity in the light of Ad-loaded MSCs and for the strategy to evade host immunity such as use of immunosuppressive agents.

\section{Abbreviations}

MSCs: Mesenchymal stem cells; Ad: Adenoviruses; CAR: Coxsackie adenovirus receptor; IFN: Interferon; IL: Interleukin; FITC: Fluorescein isothiocyanate; Ab: Antibody; GFP: Green fluorescent protein; LacZ: $\beta$-galactosidase; MOI: Multiplicity of infection; RT-PCR: Reverse transcription-polymerase chain reaction; GAPDH: Glyceraldehyde-3-phosphate dehydrogenase;

ELISA: Enzyme-linked immunosorbent assay; CMV: Cytomegalovirus.

\section{Competing interests}

The authors declare that they have no competing interests.

\section{Authors' contributions}

TS, KK, QL and SO conducted experiments, YT, HS and KH analyzed the data, KT and NY organized the experiments, NY and MT prepared the manuscript. All authors read and approved the final manuscript. 


\section{Acknowledgements}

This work was supported by Grants-in-Aid for Scientific Research from the Ministry of Education, Culture, Sports, Science and Technology of Japan, Grant-in-Aid for Research on seeds for Publicly Essential Drugs and Medical Devices from the Ministry of Health, Labor and Welfare of Japan, and a Grant-in-aid from the Nichias Corporation which does not have any patents, products in development or marketed products to declare.

\section{Sources of support}

Grants-in-Aid for Scientific Research from the Ministry of Education, Culture, Sports, Science and Technology of Japan, the Grant-in-Aid for Research on seeds for Publicly Essential Drugs and Medical Devices from the Ministry of Health, Labor and Welfare of Japan, and a Grant-in-aid from the Nichias Corporation.

\section{Author details}

'Department of Molecular Cell Biology, Graduate School of Pharmaceutical Sciences, Chiba University, Chiba, Japan. '2Division of Pathology and Cell Therapy, Chiba Cancer Center Research Institute, Chiba, Japan. ${ }^{3}$ Department of Molecular Biology and Oncology, Graduate School of Medicine, Chiba University, Chiba, Japan. ${ }^{4}$ Department of Respirology, Graduate School of Medicine, Chiba University, Chiba, Japan. ${ }^{5}$ Department of Surgery, School of Medicine, Toho University, Tokyo, Japan. ${ }^{6}$ Department of Pathology, Tokyo Women's Medical University Yachiyo Medical Center, Yachiyo, Japan.

Received: 10 September 2014 Accepted: 23 September 2014 Published: 25 September 2014

\section{References}

1. Pittenger MF, Mackay AM, Beck SC, Jaiswal RK, Douglas R, Mosca JD, Moorman MA, Simonetti DW, Craig S, Marshak DR: Multilineage potential of adult human mesenchymal stem cells. Science 1999, 284:143-147.

2. Hamada H, Kobune M, Nakamura K, Kawano Y, Kato K, Honmou O, Houkin K, Matsunaga T, Niitsu Y: Mesenchymal stem cells (MSC) as therapeutic cytoreagents for gene therapy. Cancer Sci 2005, 96:149-156.

3. Barry FP, Murphy JM: Mesenchymal stem cells: clinical applications and biological characterization. Int J Biochem Cell Biol 2004, 36:568-584.

4. Studeny M, Marini FC, Dembinski JL, Zompetta C, Cabreira-Hansen M, Bekele BN, Champlin RE, Andreeff M: Mesenchymal stem cells: potential precursors for tumor stroma and targeted-delivery vehicles for anticancer agents. J Nat/ Cancer Inst 2004, 96:1593-1603.

5. Tuve S, Wang H, Ware C, Liu Y, Gaggar A, Bernt K, Shayakhmetov D, Li Z, Strauss $R$, Stone $D$, Lieber $A$ : A new group $B$ adenovirus receptor is expressed at high levels on human stem and tumor cells. J Virol 2006, 80:12109-12120.

6. Shayakhmetov DM, Papayannopoulou T, Stamatoyannopoulos G, Lieber A: Efficient gene transfer into human $\mathrm{CD} 34(+)$ cells by a retargeted adenovirus vector. Virol 2000, 74:2567-2583.

7. Yu L, Shimozato O, Li Q, Kawamura K, Ma G, Namba M, Ogawa T, Kaiho I, Tagawa M: Adenovirus type 5 substituted with type 11 or 35 fiber structure increases its infectivity to human cells enabling dual gene transfer in CD46-dependent and -independent manners. Anticancer Res 2007, 27:2311-2316.

8. Gaggar A, Shayakhmetov DM, Lieber A: CD46 is a cellular receptor fo group B adenoviruses. Nat Med 2003, 9:1408-1412.

9. Li Q, Kawamura K, Tada Y, Shimada H, Hiroshima K, Tagawa M: Novel type III interferons produce anti-tumor effects through multiple functions. Front Biosci (Landmark Ed) 2013, 18:909-918.

10. Lasfar A, Abushahba W, Balan M, Cohen-Solal KA: Interferon lambda: a new sword in cancer immunotherapy. Clin Dev Immunol 2011, 2011:349575.

11. Fujie H, Tanaka T, Tagawa M, Kaijun N, Watanabe M, Suzuki T, Nakayama K, Numasaki M: Antitumor activity of type III interferon alone or in combination with type I interferon against human non-small cell lung cancer. Cancer Sci 2011, 102:1977-1990

12. Li Q, Kawamura K, Ma G, Iwata F, Numasaki M, Suzuki N, Shimada H, Tagawa M: Interferon- $\lambda$ induces $\mathrm{G} 1$ phase arrest or apoptosis in oesophageal carcinoma cells and produces anti-tumour effects in combination with anti-cancer agents. Eur J Cancer 2010, 46:180-190.

13. Lasfar A, Lewis-Antes A, Smirnov SV, Anantha S, Abushahba W, Tian B, Reuhl K, Dickensheets H, Sheikh F, Donnelly RP, Raveche E, Kotenko SV:
Characterization of the mouse IFN- $\lambda$ ligand-receptor system: IFN- $\lambda$ s exhibit antitumor activity against B16 melanoma. Cancer Res 2006, 66:4468-4477.

14. Sato A, Ohtsuki M, Hata M, Kobayashi E, Murakami T: Antitumor activity of IFN- $\lambda$ in murine tumor models. J Immunol 2006, 176:7686-7694.

15. Numasaki M, Tagawa M, Hidaka M, Suzuki T, Nakamura A, Okada M, Iwakura Y, Aiba S, Yamaya M: IL-28 elicits antitumor responses against murine fibrosarcoma. J Immunol 2007, 178:5086-5098.

16. Abushahba W, Balan M, Castaneda I, Yuan Y, Reuhl K, Raveche E, de la Torre A, Lasfar A, Kotenko SV: Antitumor activity of type I and type III interferons in BNL hepatoma model. Cancer Immunol Immunother 2010, 59:1059-1071.

17. Lasfar A, Cohen-Solal KA: Emergence of IFN-lambda as a potential antitumor agent. In Targets in Gene Therapy. 15th edition. Edited by You YP. Croatia: InTech; 2011

18. Fushimi K, lijima M, Gao C, Kondo T, Tsuji T, Hashimoto T, Mihara K, Namba M: Transformation of normal human fibroblasts into immortalized cells with the mutant p53 gene and X-rays. Int J Cancer 1997, 70:135-140.

19. Compton T: An immortalized human fibroblast cell line is permissive for human cytomegalovirus infection. J Virol 1993, 67:3644-3648.

20. Miyauchi M, Yoshida Y, Tada Y, Narita M, Maeda T, Bahar R, Kadomatsu K, Muramatsu T, Matsubara S, Nakagawara A, Sakiyama S, Tagawa M: Expression of herpes simplex virus-thymidine kinase gene controlled by a promoter region of the midkine gene confers selective cytotoxicity to ganciclovir in human carcinoma cells. Int J Cancer 2001, 91:723-727.

21. Kawamura K, Yu L, Tomizawa M, Shimozato O, Ma G, Li Q, Sato A, Yang Y, Suzuki T, Abdel-Aziz NM, Tagawa M: Transcriptional regulatory regions of the survivin gene activate an exogenous suicide gene in human tumors and enhance the sensitivity to a prodrug. Anticancer Res 2007, 27:89-93.

22. Yamamoto M, Alemany R, Adachi Y, Grizzle WE, Curiel DT: Characterization of the cyclooxygenase-2 promoter in an adenoviral vector and its application for the mitigation of toxicity in suicide gene therapy of gastrointestinal cancers. Mol Ther 2001, 3:385-394.

23. Lyle C, McCormick F: Integrin av $\beta 5$ is a primary receptor for adenovirus in CAR-negative cells. Virol J 2010, 7:148

24. Murakami S, Sakurai F, Kawabata K, Okada N, Fujita T, Yamamoto A, Hayakawa T, Mizuguchi H: Interaction of penton base Arg-Gly-Asp motifs with integrins is crucial for adenovirus serotype 35 vector transduction in human hematopoietic cells. Gene Ther 2007, 14:1525-1533.

25. Knaän-Shanzer S, van de Watering MJ, van der Velde I, Gonçalves MA Valerio D, de Vries AA: Endowing human adenovirus serotype 5 vectors with fiber domains of species B greatly enhances gene transfer into human mesenchymal stem cells. Stem Cells 2005, 23:1598-1607.

26. Seo SH, Kim KS, Park SH, Suh YS, Kim SJ, Jeun SS, Sung YC: The effects of mesenchymal stem cells injected via different routes on modified IL-12mediated antitumor activity. Gene Ther 2011, 18:488-495.

27. Wang GX, Zhan YA, Hu HL, Wang Y, Fu B: Mesenchymal stem cells modified to express interferon- $\beta$ inhibit the growth of prostate cancer in a mouse model. J Int Med Res 2012, 40:317-327.

28. Hakkarainen T, Särkioja M, Lehenkari P, Miettinen S, Ylikomi T, Suuronen R, Desmond RA, Kanerva A, Hemminki A: Human mesenchymal stem cells lack tumor tropism but enhance the antitumor activity of oncolytic adenoviruses in orthotopic lung and breast tumors. Hum Gene Ther 2007. 18:627-641.

29. Yong RL, Shinojima N, Fueyo J, Gumin J, Vecil GG, Marini FC, Bogler O, Andreeff M, Lang FF: Human bone marrow-derived mesenchymal stem cells for intravascular delivery of oncolytic adenovirus Delta24-RGD to human gliomas. Cancer Res 2009, 69:8932-8940.

30. García-Castro J, Alemany R, Cascalló M, Martínez-Quintanilla J, Arriero Mdel M Lassaletta A, Madero L, Ramírez M: Treatment of metastatic neuroblastoma with systemic oncolytic virotherapy delivered by autologous mesenchymal stem cells: an exploratory study. Cancer Gene Ther 2010, 17:476-483.

31. Hamada K, Desaki J, Nakagawa K, Zhang T, Shirakawa T, Gotoh A, Tagawa M: Carrier cell-mediated delivery of a replication-competent adenovirus for cancer gene therapy. Mol Ther 2007, 15:1121-1128.

32. Yu L, Hamada K, Namba M, Kadomatsu K, Muramatsu T, Matsubara S, Tagawa M: Midkine promoter-driven suicide gene expression and -mediated adenovirus replication produced cytotoxic effects to immortalised and tumour cells. Eur J Cancer 2004, 40:1787-1794.

33. Ono HA, Davydova JG, Adachi Y, Takayama K, Barker SD, Reynolds PN, Krasnykh VN, Kunisaki C, Shimada H, Curiel DT, Yamamoto M: Promotercontrolled infectivity-enhanced conditionally replicative adenoviral vectors for the treatment of gastric cancer. J Gastroentero/ 2005, 40:31-42. 
34. Zhu ZB, Chen Y, Makhija SK, Lu B, Wang M, Rivera AA, Yamamoto M, Wang S, Siegal GP, Curiel DT, McDonald JM: Survivin promoter-based conditionally replicative adenoviruses target cholangiocarcinoma. Int J Oncol 2006, 29:1319-1329.

35. Yu L, Ugai S, O-Wang J, Namba M, Kadomatsu K, Muramatsu T, Matsubara S, Sakiyama S, Tagawa M: Cell growth- and p53-dependent transcriptional activity of the midkine promoter confers suicide gene expression in tumor cells. Oncol Rep 2003, 10:1301-1305.

36. Burke SJ, Collier JJ: The gene encoding cyclooxygenase-2 is regulated by IL-1 $\beta$ and prostaglandins in 832/13 rat insulinoma cells. Cell Immunol 2011, 271:379-384.

37. Ulivi V, Giannoni P, Gentili C, Cancedda R, Descalzi F: p38/NF-kB-dependent expression of COX-2 during differentiation and inflammatory response of chondrocytes. J Cell Biochem 2008, 104:1393-1406.

doi:10.1186/1471-2407-14-713

Cite this article as: Suzuki et al:: Mesenchymal stem cells are efficiently transduced with adenoviruses bearing type 35-derived fibers and the transduced cells with the IL-28A gene produces cytotoxicity to lung carcinoma cells co-cultured. BMC Cancer 2014 14:713.

\section{Submit your next manuscript to BioMed Central and take full advantage of:}

- Convenient online submission

- Thorough peer review

- No space constraints or color figure charges

- Immediate publication on acceptance

- Inclusion in PubMed, CAS, Scopus and Google Scholar

- Research which is freely available for redistribution 